\title{
Electronic Structure of MoSe2/Graphene Van der Waals Heterostructures: an ab-initio Study
}

\author{
1,* Weverson Lucas Aguiar (PG), ${ }^{1}$ Bernhard Georg Enders Neto (PQ) and ${ }^{2}$ Luiz Antônio \\ Ribeiro Júnior (PQ) \\ ${ }^{1}$ University of Brasília, PPG-CIMA, Campus Planaltina, 73345-010, Brasília, DF, Brazil, ${ }^{2}$ International \\ Center for Condensed Matter Physics, University of Brasília, P.O. Box 04531, 70.919-970, Brasília, DF, \\ Brazil \\ Keywords: Graphene, MoSe2, Van der Waals, Heterojuntions.
}

\section{Introduction}

Two-dimensional layered Transition Metal Dichalcogenides (TDMs), especially MoS2, are promising materials for developing a new class of optoelectronic devices due to their high photosensitivity, which is the result of its indirect to direct band gap transition when the bulk dimension is reduced to a single monolayer. using a commercial software package named Materials Studio, which is developed by Accelrys Inc. This software includes the modules DMol3 and CASTEP, that use DFT with a numerical radial function basis and with a plane wave basis set. We theoretically investigate the electronic structure of a van der Waals heterojuntions formed between single layers of MoSe2 and graphene. The present study may shedding light on essential features such as doping, Fermi velocity, hybridization, and band-offset of the low energy electronic dynamics found at the interface.

\section{Methodology}

The DFT calculations presented here were carried out using a commercial software package named Materials Studio, which is developed by Accelrys Inc. This software includes the modules DMol3 and CASTEP, that use DFT with a numerical radial function basis and with a plane wave basis set, respectively, to calculate the electronic properties of molecules, clusters, surfaces and crystalline solid materials from first principles. Here we calculate the electronic and optical properties of MoSe2/Graphene heterojuctions by using the methods LDA/PW91 and GGA/PBE on DFT, as implemented in the modules CASTEP and DMol3.

\section{Results}

Based on the DFT calculations, we investigate the electronic band structures of MoSe2/Graphene heterojunctions. The results show that there is a non-uniform strain between two different orientation angles of $\mathrm{MoSe} 2$ monolayer on top of epitaxial bilayer graphene. Hence, our results show that strain-induced bandgap engineering of single layered $\mathrm{MoSe} 2$ is dependent on the orientation angle between stacked layers. This finding could be an interesting novel way to take advantage of the possibilities of 
MoSe2 and deeply exploit the capabilities of TMDs/graphene van der Waals heterostructures.

\section{Conclusions}

To conclude, we have reported that the orientation of MoSe 2 monolayer on bilayer graphene heterostructures affects the bandgap of MoSe2. This suggests the dependency of the bandgap of layered TMD structures on the interfacial interactions within the heterostructures.

\section{Acknowledgements}

The authors gratefully acknowledge the financial support from the Brazilian Research Councils CAPES and FAPDF. L.A.R.J. gratefully acknowledges the financial support from the Brazilian Research Council FAPDF grant 0193.000942/2015 and 193.001.511/2017. L.A.R.J. also wishes to thank the Brazilian Ministry of Planning, Budget and Management (Grant DIPLA 005/2016).

\section{References}

[1] M. M. Ugeda et. al. Nature Materials, 13, 1091-1095, 2014.

[2] Z. B. Aziz et. al. Carbon, 110, 396-403, 2016. 